\title{
Special issue on power amplifier linearization
}

\author{
JOHN WOOD ${ }^{1}$ AND EDUARD BERTRAN ${ }^{2}$
}

We are honored to be the guest editors of this Special Issue on linearization and modeling of RF power amplifiers (PAs). In recent years, there has been an upsurge in interest in the behavioral modeling of high-power RF amplifiers, and applying these modeling techniques to the linearization of the PAs using pre-distortion methods, usually applied at baseband frequencies, in the digital domain: hence digital pre-distortion or DPD. This increased interest has been evident in several workshops and focus sessions at the International Microwave Symposium, the European Microwave Conference, and the Radio and Wireless Symposium in recent years. To our knowledge, this is the first special issue of a learned journal devoted to the behavioral modeling and linearization of RF PAs.

Linearization as a topic is well established, in many fields of endeavor. Notably mathematical in nature, and often in pure form, it is often abstracted from a real application. But here we have a very practical use for the nonlinear mathematics, so it is therefore not a surprise that most of the tools for the linearization of PAs are found in the mathematics of nonlinear dynamical systems, and applied specifically to this interesting problem. Of significant importance, PAs are the backbone of wireless telecommunications systems. They are also the most power hungry devices among the transmitter subsystems. To reduce the power consumption, or, alternatively, to reduce the distortion produced by the PA, not only benefits directly billions of people worldwide using mobile communications by increasing the time of life of their batteries, but also shrinks the bill of the energy consumed in base-stations. This is achieved not only by improving the energy efficiency of the PA itself but also by reducing or removing the need for air conditioning systems in the base-stations, again reducing the energy costs and the carbon footprint of the base station: the behavioral modeling and linearization of PAs support a greener environment.

As the demand for increased data over wireless continues to increase, the signal bandwidth, spectral efficiency, and modulation complexity have also increased, and this has raised the interest in PA linearizers significantly, making predistortion a commercial reality. The continued increase in signal bandwidth to cope with this increased data requires a similar increase in the bandwidth of the linearizer, and has

\footnotetext{
${ }^{1}$ Maxim Integrated, San Jose, California, USA

${ }^{2}$ Universitat Politècnica de Catalunya, Barcelona, Catalonia, Spain Corresponding author:

John Wood and Eduard Bertran

Emails: john.wood@maximintegrated.com; bertran@tsc.upc.edu
}

promoted research on different technologies and strategies to overcome these bandwidth demands and produce more compact yet effective modeling and linearizing schemes. Such approaches see compromises between bandwidth, memory depth, and overall model complexity to achieve optimum model accuracy and a level of linearization that is sufficient for the given practical application.

In this Special Issue of IJMWT, we bring together a collection of papers detailing various aspects of modeling and linearization, from a review of recent model developments and characterization and test methods, to new approaches for model parameter identification and implementation in DPD systems, in the face of the many and varied challenges to deliver more voice and data information, without compromising the quality of service. Such challenges, what is new in PA linearization, and the inventive and robust techniques for defeating them, form the thread of this issue.

There are several papers that focus on the fundamentals of the behavioral modeling of RF PAs, with the goal being to produce compact yet effective DPD algorithms. The papers of Braithwaite [1] and Boumaiza [2] both address the issue of determining the appropriate memory description for the PA. Braithwaite analyses the sampling rate requirements, using a Gaussian channel model to make the mathematical treatment tractable, enabling the number and separation of the delay taps to be traded off against the model accuracy to achieve a practical PA model, and hence a pre-distorter that is compact and effective. Boumaiza employs the mathematical method of embedding to determine the memory tap spacing and memory depth, using a variety of techniques to estimate memory characteristic. Again, a compact and effective DPD linearizer is constructed and demonstrated. The paper by Mehdi et al. [3] applies model-order reduction techniques such as Akaike and Bayesian information criteria to reduce the number of model parameters in a systematic manner, again trading off model complexity against accuracy to arrive at the optimum model. This approach has been applied to build pre-distorters for traditional single inputsingle-output PAs and also multiple input-multiple output (MIMO) PA systems. In Sombrin's paper [4], non-analytic functions that are invariant elements of the Chebyshev Transform are used to generalize the polynomial nonlinearity theory, producing models that exhibit intermodulation behavior significantly different from that of classical polynomial models, giving theoretical foundation for nonlinearities such as threshold and hard limiter functions. The final modeling paper is by Maziere et al. [5], who used a different approach 
from the time-domain functional modeling described in the preceding papers. Here, a frequency domain model that is an extension of the multi-harmonic X-parameters model to include memory effects using a Volterra-based model is described, as an approach to the behavioral modeling of PAs.

The remaining five papers address some of the current challenges that are presented in the world of PA linearization, and also describe some of the more practical aspects. Guan [6] presents a design procedure for implementing a flexible FPGA-based DPD platform that includes an analog RF front-end, a digital baseband unit, and a system control panel. This platform can be used to evaluate various DPD algorithms for different types of RF PAs. The second paper of Braithwaite [7] presents a contemporary problem in the design of DPD linearizers for new communication standards because of their wide bandwidth. It proposes a solution for DPD designs when the observation bandwidth is narrower than the distortion spectrum. The other three papers all demonstrate the application of pre-distortion techniques to Envelope-Tracking (ET) PAs. Yan [8] considers an ET system under the operating conditions of reduced power demand from the PA, and presents a pre-distorter that effectively scales with the power demand. In the paper by McCallister [9], a combination of signal peak reduction techniques and a simple pre-distortion method to compensate some physical issues that generate memory effects are presented. These are applied to the linearization of an ET PA in integrated system architecture. Gilabert and Montoro [10] present two computationally efficient approaches for designing a DPD system suitable for ET PAs, and consider the practical implications of implementing these algorithms in FPGA devices.

As guest editors, we are grateful to the authors for their contributions and to the reviewers for their diligence and constructive comments and recommendations. We would also like to thank editors-in-chief of this journal, Professor Quere and Professor Suarez, for their help and guidance through the editorial process, and to the journal editorial assistant, Amanda Johns, for shepherding the papers through to publication.

\section{REFERENCES}

[1] Neil Braithwaite, R.: Sampling requirements for nonlinear basis waveforms used in digital predistortion and amplifier modeling. Int. J. Microwave Wireless Technol., 5 (2) (2013), 103-113.

[2] Fehri, B.; Boumaiza, S.: Application of embedding dimension estimation to Volterra series based behavioral modeling and predistortion of wideband RF power amplifier. Int. J. Microwave Wireless Technol., 5 (2) (2013), 115-122.

[3] Amiri, M.V.; Bassam, S.A.; Helaoui, M.; Ghannouchi, F.M.: New order selection technique using information criteria applied to SISO and MIMO systems predistortion. Int. J. Microwave Wireless Technol., 5 (2) (2013), 123-131.

[4] Sombrin, J.: Non-analytic behavioural models for active or passive nonlinearity. Int. J. Microwave Wireless Technol., 5 (2) (2013), 133-140.

[5] Maziere, C.; Gatard, E.; Enguehard, C.; Gustavsen, B.: A multiharmonic model taking into account coupling effects of long and short-term memory in SSPAs. Int. J. Microwave Wireless Technol., 5 (2) (2013), 141-148.
[6] Guan, L.; Kearney, R.; Yu, C.; Zhu, A.: High performance digital predistortion test platform development for wideband RF power amplifiers. Int. J. Microwave Wireless Technol., 5 (2) (2013), 149-162.

[7] Neil Braithwaite, R.: Model order selection for digital predistortion of a RF power amplifier when the distortion spectrum exceeds the observation bandwidth. Int. J. Microwave Wireless Technol., 5 (2) (2013), 163-170.

[8] Yan, J.J.; Draxler, P.; Presti, C.; Kimball, D.; Asbeck, P.M.: Digital predistortion of envelope tracking power amplifiers under average power back-off and long-term average power efficiency for basestation applications. Int. J. Microwave Wireless Technol., 5 (2) (2013), 171-177.

[9] McCallister, R.: Ideal amplification of broadband signals. Int. J. Microwave Wireless Technol., 5 (2) (2013), 179-186.

[10] Gilabert, P.L.; Montoro, G.: Computationally efficient real-time digital predistortion architectures for envelope tracking power amplifiers. Int. J. Microwave Wireless Technol., 5 (2) (2013), 187-193.

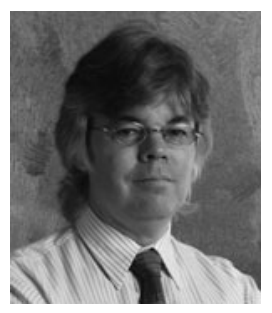

John Wood received B.Sc. and Ph.D. degrees in Electrical and Electronic Engineering from the University of Leeds, in 1976 and 1980 , respectively. He is currently Senior Principal Member of Technical Staff with Maxim Integrated, working on the system architecture of digital pre-distortion (DPD) solutions. He was a Distinguished Member of the Technical Staff in the RF Division of Freescale Semiconductor, where he worked from 2005 to 2011 . His areas of expertise include the development of nonlinear compact device models and behavioral models for RF power transistors and ICs, the understanding of the impact, characterization, and control using DPD of nonlinearities and memory effects in high-efficiency PAs. He is author or co-author of over 130 papers and articles. $\mathrm{He}$ is a Fellow of the IEEE, and a member of the Microwave Theory and Techniques, and Electron Devices Societies, and is a member of ARFTG Executive Committee. He is a Distinguished Microwave Lecturer for MTT Society. He is currently Editor-in Chief of the IEEE 'Microwave' magazine.

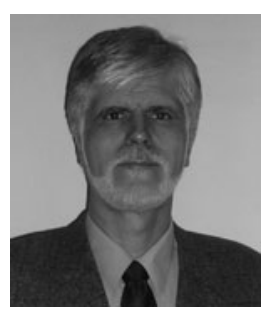

Eduard Bertran received the Engineer and Doctor Engineer degrees in telecommunication from the Technical University of Catalonia (UPC), Barcelona, Spain, in 1979 and 1985, respectively. In 1987, he joined the Department of Signal Theory and Communications (TSC), UPC, where he is currently a Full Professor. He has been the Head of Studies of the Department, Coordinator of some Doctorate Programs and Curricula Director in Telecommunication Schools. His research interests include Control, Communication Electronics, Signal Processing, and Circuit Theory. He has collaborated in different national and European research projects and teams, and he has also collaborated in different industrial projects (he held a patent on RF amplifiers). He is author or co-author of a number of journal and conference papers, as well as books and book chapters. During last decade he has worked on the PA linearization problem, by using both analog and digital approaches. He is a Senior Member of IEEE. 